\title{
Palliative radiotherapy in advanced cancer patients treated with immune-checkpoint inhibitors: The PRACTICE study
}

\author{
MELISSA BERSANELLI $^{1,2}$, ELISABETTA LATTANZI ${ }^{3}$, NUNZIATA D'ABBIERO ${ }^{3}$, SEBASTIANO BUTI $^{2}$, \\ ALESSANDRO LEONETTI ${ }^{2}$, MARIA GIULIA CANE ${ }^{3}$, SALVATORE TRAPANI ${ }^{2}$, GIOVANNI LUCA GRAVINA ${ }^{4}$, \\ GIAMPIERO PORZIO $^{4,5}$, KATIA CANNITA ${ }^{5}$, PIETRO DI MARINO ${ }^{6}$, ANTONINO GRASSADONIA ${ }^{7}$, \\ NICOLA TINARI ${ }^{7}$, MICHELE DE TURSI ${ }^{7}$, ELISA GIAIACOPI $^{2}$, MARIA MICHIARA $^{2}$, PAOLA BORDI $^{2}$, \\ FABIANA PERRONE ${ }^{2}$, LUCIANA CARAVATTA $^{8}$, MARIANNA TRIGNANI $^{8}$, DOMENICO GENOVESI $^{8}$, \\ CLARA NATOLI $^{7}$, CORRADO FICORELLA $^{4,5}$, MARCELLO TISEO $^{1,2}$ and ALESSIO CORTELLINI ${ }^{4,5}$ \\ ${ }^{1}$ Department of Medicine and Surgery, ${ }^{2}$ Medical Oncology Unit, ${ }^{3}$ Radiotherapy Unit, University Hospital of Parma, \\ I-43126 Parma; ${ }^{4}$ Department of Biotechnological and Applied Clinical Sciences, University of L'Aquila; \\ ${ }^{5}$ Medical Oncology Unit, St. Salvatore Hospital, I-67100 L'Aquila; ${ }^{6}$ Clinical Oncology Unit, \\ Santissima Annunziata Hospital; ${ }^{7}$ Department of Medical, Oral and Biotechnological Sciences, \\ Gabriele D'Annunzio University of Chieti-Pescara; ${ }^{8}$ Department of Radiation Oncology, \\ Santissima Annunziata Hospital, Gabriele D'Annunzio University of Chieti-Pescara, I-66100 Chieti, Italy
}

Received September 10, 2019; Accepted November 6, 2019

DOI: $10.3892 / b r .2019 .1265$

\begin{abstract}
In the present study, the influence of purely palliative radiotherapy (pRT) on the outcomes of patients with advanced cancer undergoing immune checkpoint blockade was evaluated. Patients were stratified into three groups: Patients who had received pRT within 6 months prior to the initiation of immunotherapy (previous pRT); patients who received pRT during immunotherapy (concurrent pRT); and patients who did not receive RT prior to or during immunotherapy (no RT group), and these groups were compared. The median overall survival (mOS), median progression free survival (mPFS) and median time-to-treatment failure (mTTF) for the previous pRT group were significantly shorter compared with the no RT group (mOS, 3.6 vs. 12.1 months, respectively, $\mathrm{P}=0.0095$; mPFS 1.8 vs. 5.4 months, respectively, $\mathrm{P}=0.0016$; mTTF 1.8 vs. 5.7 months, respectively, $\mathrm{P}=0.0035$ ). The concurrent pRT group had a longer mTTF compared with the previous pRT group and similar outcomes to the no RT group. In the previous pRT group, $26.9 \%$ of the patients experienced immune-related adverse events compared with $40.1 \%$ of patients in the no RT group. Despite the use of pRT during immunotherapy being considered safe, the results of the present study suggest that pRT has a negative effect on immune balance.
\end{abstract}

Correspondence to: Dr Alessio Cortellini, Medical Oncology Unit, St. Salvatore Hospital, 48 Via Vetoio, I-67100 L'Aquila, Italy E-mail: alessio.cortellini@graduate.univaq.it

Key words: palliative radiotherapy, immunotherapy, immune checkpoint inhibitors, anti-PD-1, anti-PD-L1, abscopal effect, immunosuppression

\section{Introduction}

As the use of immunotherapy for treating various types of cancer becomes more widespread, several issues require investigation to determine their possible impact on the outcome of cancer patients treated with anti-PD-1/PD-L1 immune checkpoint inhibitors (CKIs) (1). Thus, the respective effects of concomitant medications, concurrent treatments and other possible immunomodulatory events in the clinical history of patients prior to the initiation of immunotherapy, or during its course, have been largely explored in the recent years, obtaining a wide range of controversial evidence (1-5). For example, it seems that the use of corticosteroids or antibiotics before or with CKIs may decrease efficacy of the latter, whereas the use of influenza vaccine may be beneficial irrespective of its anti-infectious efficacy (2-4). Amongst all the topics explored in relation to immunotherapy, radiotherapy (RT) is a considerably important issue, since the interest in the abscopal effect has recently been rediscovered, and described in relation to immunotherapy (6). The abscopal effect of local $\mathrm{RT}$ is considered as a systemic anti-tumor immune response which reflects the regression of non-irradiated metastatic lesions at a distance from the primary site of irradiation (6). The relationship between the abscopal effect and the immune system, particularly regarding lymphocytes, has been known since 1969 (7). As long as the immune checkpoint blockade has been used to enhance the immune response, their possible synergy with RT has been investigated (6,7). Although the exact underlying mechanism of the abscopal effect remains unclear, the administration of CKIs can enhance the anti-tumor immunogenicity of RT, by preventing PD-1/PD-L1 induced T cell anergy (6).

Nevertheless, the abscopal effect is known to be uncommon and RT is generally unable to subvert the immune tolerance 
towards the tumor (8). Combination of RT with anticancer immunotherapy aims to shift the balance of the immunosuppressive tumor microenvironment to achieve tumor rejection, inducing the positive effects of RT to overcome the possible negative effects.

Retrospective studies have been performed to investigate the potential effect of RT when administered before or during immunotherapy. Kiess et al (9) evaluated 46 patients with 113 brain metastases from a melanoma, who were treated with ipilimumab and single-fraction stereotactic radiosurgery (SRS) together, and found that patients treated with SRS before or during administration of ipilimumab had significantly improved overall survival (OS) and good local disease control after 1 year compared with patients treated with SRS following treatment with ipilimumab (9). Following retrospective studies examining the effects of anti-CTLA-4 CKIs (9), other retrospective reports regarding stereotactic RT/SRS during immunotherapy confirmed its likely positive interaction with anti-PD-1/PD-L1 CKIs (10). Furthermore, a recent randomized prospective trial, assessed whether stereotactic RT on a single tumor site prior to treatment with pembrolizumab treatment enhanced tumor response in patients with metastatic non-small cell lung cancer (NSCLC), and demonstrated a doubling of objective response rates (ORR) with RT immediately prior to immunotherapy compared with pembrolizumab alone, although the results did not meet the study's prespecified end point criteria for meaningful clinical benefit (11).

A secondary retrospective analysis of a subset of patients treated with the anti-PD-1 pembrolizumab in the prospective phase 1 KEYNOTE-001 study was performed by Shaverdian et al (12). They found that patients who had previously (at any time) received RT for the treatment of NSCLC, before the initiation of systemic treatment with pembrolizumab, had significantly longer survival, compared with patients who had not received previous RT (12). This study currently represents the largest clinical evidence about the effect of previous RT on the outcome of patients to immunotherapy, although such data need to be furtherly validated with prospective trials.

Together, previous studies have highlighted the possibility of triggering an abscopal effect, particularly in cases with high-dose low-volume RT. To investigate the effect of different types of RT used in clinical practice, a Palliative Radiotherapy in Advanced Cancer patients Treated with Immune- $C \mathrm{~h} E$ ckpoint inhibitors (PRACTICE) retrospective analysis was performed, to compare the clinical outcome of patients who underwent palliative RT (pRT) prior to the initiation of anti-PD-1/PD-L1 CKIs, with patients who did not receive RT or patients receiving pRT during the course of immunotherapy.

\section{Patients and methods}

Patients. The present study included patients with advanced cancer with histologically confirmed diagnosis of a tumor of any primary origin, whom consecutively underwent treatment with single agent anti-PD-1/PD-L1, regardless of the treatment line, at the Medical Oncology Departments of three Italian centers, the University Hospital of Parma (Parma, Italy), University Hospital of L'Aquila (L,Auila, Italy) and the University Hospital of Chieti (Chieti, Italy), between August 2015 and September 2017. A total of 192 patients with advanced cancer were recruited for the present study and their median age was 68.6 years (range, 32-87), with 143 male and 49 female participants. Patients were stratified according to whether they received $\mathrm{pRT}$, received $\mathrm{pRT}$ prior to immunotherapy, or received pRT during immunotherapy. The administration of high-dose RT for non-palliative use was an exclusion criterion. All patients provided written, informed consent for treatment with immunotherapy.

Study design. A multicenter retrospective analysis of patients with advanced cancer treated with anti-PD-1/PD-L1 CKIs, regardless of treatment line, was performed. The primary aim of the present analysis was to compare the clinical outcomes of patients who received pRT prior to immunotherapy (6-months before initiation of immunotherapy) compared with patients who did not receive any RT within 6 months prior to initiation of immunotherapy, or during immunotherapy. The ORR, disease control rate (DCR), progression free survival (PFS) in months, time to treatment failure (TTF) in months, OS in months, and rate of immune-related adverse events (irAEs) were compared between the groups.

In order to overcome a potential positive selection bias of patients who did not require RT in their clinical history, the secondary aim of the study was to compare the clinical outcomes of patients who received pRT within 6 months prior to immunotherapy initiation and patients who received $\mathrm{pRT}$ during immunotherapy, in terms of ORR, PFS, TTF, OS and rate of irAEs.

ORR was defined as the proportion of patients who experienced an objective response (complete response or partial response) as the best response to immunotherapy according to RECIST criteria, version 1.1 (13). DCR was defined as the proportion of patients who experienced an objective response or stabilization of the disease as the best response to treatment. TTF was defined as the time from treatment's start to discontinuation for any reason, including disease progression, treatment toxicity, patient preference or death. PFS was defined as the time from CKI treatment initiation to documented disease progression or death, or to the last contact for alive patients. OS was calculated as the time from the beginning of CKI treatment and death, or to the last contact for alive patients.

The following covariates were analyzed: Primary tumor (NSCLC, melanoma, renal cell carcinoma, others), age ( $<70$ years vs $\geq 70$ years old, based on previous studies) (14-16), sex (male vs. female), Eastern Cooperative Oncology Group Performance Status (ECOG PS; 0-1 vs. $\geq 2$ ) (17), treatment line (first vs. further lines), presence of bone metastases (yes vs. no, defined as 'baseline bone metastases') and presence of central nervous system (CNS) metastases (yes vs. no, defined as 'baseline CNS metastases') at baseline before immunotherapy.

IrAEs were graded according to the National Cancer Institute Common Toxicity Criteria for Adverse Events (version 5.0) (18) and cumulatively reported as crude incidence.

Radiotherapy. The time-window for defining 'previous pRT' was set at 6-months before initiation of immunotherapy, in 
accordance with an arbitrary choice of the investigators, as it was determined to be a reasonable compromise between the fact that events too far from the beginning of therapy may not have an impact on treatment outcome, and the well-demonstrated long-lasting effect of RT on the immune system $(19,20)$.

Patients who had received $\mathrm{RT}>6$ months prior to immunotherapy initiation and patients who received RT after permanent discontinuation of immunotherapy were included in the control group, together with patients who had never received RT. Patients receiving a high-dose of RT for non-palliative reasons were excluded from the present study.

Palliative RT treatments were performed (including stereotactic RT or SRS) according to the clinical practice of the participating centers and were defined as conventional radiation therapy administered without curative intent, for the local control of symptoms to metastatic sites of advanced tumors. RT was categorized according to the treated organ/region as follows: CNS, bone, lymph-node, visceral and other. Dose (Gy) and duration were collected, with a median dose of 20 Gy and a mean dose of $23 \mathrm{~Gy}$; the dose range was 8-40 Gy. Patients were categorized into three groups according to the timing of RT, as follows: patients who had received pRT within 6 months prior to the initiation of immunotherapy (previous pRT), patients who received pRT during immunotherapy (concurrent pRT) and patients who did not receive RT prior to or during immunotherapy (no RT group).

Statistical analysis. $\chi^{2}$ and Fisher's exact test were used to evaluate ORR, DCR and the incidence of irAEs among the groups, according to the sample size in contingency tables for each comparison (21-23). Given the well-known poor prognostic impact of CNS metastases and bone metastases $(24,25)$, the differences among subgroups according to such characteristics were evaluated with a $\chi^{2}$ and Fisher's exact test, respectively.

In the multivariate analysis, logistic regression was used to evaluate the parameters which were significantly different in the univariate analysis of DCR (26). Median PFS (PFS) and median OS (OS) were evaluated using the Kaplan-Meier method (27). Median follow-up was calculated according to the reverse Kaplan-Meier method (28). Cox proportional hazard models were used to evaluate predictive variables in the univariate and multivariate analysis for median TTF (TTF) and mOS as described previously (29). The data cut-off period was set as September 2018. All statistical analyses were performed using MedCalc Statistical Software version 18.6 (MedCalc Software bvba).

\section{Results}

Patient characteristics. A total of 192 patients with advanced cancer were included in the present analysis. Their characteristics are summarized in Table I. The primary tumors reported in patients were: NSCLC, 118 patients (61.4\%); melanoma, 38 patients $(19.8 \%)$, renal cell carcinoma, 23 patients $(12 \%)$; and others, 13 patients $(6.8 \%)$.

A total of 26 patients had received pRT within 6 months prior to initiation of immunotherapy $(13.5 \%)$ and were classified as previous pRT; 29 patients $(15.1 \%)$ received pRT during the course of immunotherapy and were classified as concurrent pRT; 137 patients (71.4\%) were classified as no RT (Table I).

In the previous $\mathrm{pRT}$, concurrent $\mathrm{pRT}$ and no RT groups, $18(69.2 \%), 14(48.3 \%)$ and $36(26.3 \%)$ patients had baseline bone metastases, and 4 (15.4\%), 9 (30\%) and $17(12.4 \%)$ patients had baseline CNS metastases, respectively (Table I). The incidence of bone metastases at baseline was significantly higher in the previous pRT group compared with the no-RT groups $(\mathrm{P}<0.0001)$ and the concurrent pRT group $(\mathrm{P}=0.0193)$. The incidence of CNS metastases at baseline was significantly higher in the concurrent pRT group compared with the no RT group $(\mathrm{P}=0.0124)$, whereas no significant difference was observed between the previous pRT group and the other groups in regard to CNS metastases (Table I).

Table II summarizes the characteristics of patients who received RT. Among the 26 patients in the previous pRT group, a total of 27 pRT treatments were performed, 15 (55.6\%) of which were for bone metastases. The median dose of RT treatments was 20 Gy and the mean dose was 23 Gy (range, 8-40). Among the 29 patients in the concurrent RT group, a total of 36 treatments were performed, 21 (58.4\%) of which were for bone metastases, with a median dose of $8 \mathrm{~Gy}$ and a mean dose of 14 Gy (range, 8-40).

Treatment outcome. Among the 177 evaluable patients, 50 showed partial response and 33 had stable disease; ORR was $28.2 \%$ [95\% confidence interval (CI), 20.9-37.2] and DCR was $46.9 \%$ (95\% CI, 37.3-58.1) in the overall population (data not shown).

At the median follow-up of 20.3 months, mOS for the overall population was 9.4 months $(95 \% \mathrm{CI}, 6.7-12.4$; 68 patients censored), median PFS was 4.3 months (95\% CI, 3.4-5.6; 23 patients censored) and median TTF was 5.0 months (95\% CI, 3.9-6.1; 16 patients censored). In the overall population, 67 patients $(34.9 \%)$ experienced irAEs of any grade (data not shown).

Comparisons between the previous $p R T$ and no RT groups. Among the patients in the previous pRT group, ORR to immunotherapy was $18.2 \%$ (95\% CI, 4.9-46.5; 4 responses out of 22 evaluated patients), whereas in the no RT group it was $32.3 \%$ (95\% CI, 23.1-43.8; 41 responses out of 127 evaluated patients) and the difference was not significant $(\mathrm{P}=0.2173)$. The DCR was significantly higher in the no RT group compared with the previous pRT group (52.8\% vs. $18.2 \%, \mathrm{P}=0.0026)$. Multivariate analysis confirmed the significantly higher DCR among patients who did not receive RT $(\mathrm{P}=0.0477)$. Table SI summarizes the univariate and multivariate analyses for DCR according to different patient characteristics. Baseline bone metastases and ECOG PS were significantly associated with DCR both in the univariate and multivariate analyses, whereas CNS metastases were not.

The median OS of the no RT group was 12.1 months (95\% CI, 8.1-16.5), compared with 3.6 months (95\% CI, 2.05-7.2) in the previous pRT groups (Fig. 1A), which was significantly shorter $(\mathrm{HR}=1.94 ; 95 \% \mathrm{CI}, 1.17-3.22 ; \mathrm{P}=0.0095)$. The statistical significance of this difference was not confirmed in the multivariate analysis $(\mathrm{HR}=1.64 ; 95 \% \mathrm{CI}, 0.94-2.86 ; \mathrm{P}=0.0775)$, 
Table I. Patient characteristics.

\begin{tabular}{|c|c|c|c|c|}
\hline Characteristics & Overall population & No RT & Previous pRT & Concurrent pRT \\
\hline \multicolumn{5}{|l|}{ Age, years } \\
\hline Median & 68.6 & 6971 & 67 & \\
\hline Range & $32-87$ & $32-87$ & $41-85$ & $43-83$ \\
\hline Elderly, $\geq 70$ years old $(\%)$ & $88(45.8)$ & $66(48.2)$ & $14(53.8)$ & $8(30.8)$ \\
\hline Number of patients $(\%)$ & $192(100)$ & $137(71.4)$ & $26(13.5)$ & $29(15.1)$ \\
\hline \multicolumn{5}{|l|}{$\operatorname{Sex}(\%)$} \\
\hline Male & $143(74.5)$ & $104(75.9)$ & $18(69.2)$ & $21(72.4)$ \\
\hline Female & $49(25.5)$ & $33(24.1)$ & $8(30.8)$ & $8(27.6)$ \\
\hline \multicolumn{5}{|l|}{ ECOG PS (\%) } \\
\hline $0-1$ & $149(77.6)$ & $110(80.3)$ & $17(65.4)$ & $22(75.9)$ \\
\hline$\geq 2$ & $43(22.4)$ & $27(19.7)$ & $9(34.6)$ & $7(24.1)$ \\
\hline \multicolumn{5}{|l|}{ Primary tumor $(\%)$} \\
\hline NSCLC & $118(61.4)$ & $82(59.8)$ & $18(69.2)$ & $18(62.1)$ \\
\hline Melanoma & $38(19.8)$ & $30(21.9)$ & $1 \quad(3.9)$ & $7(24.1)$ \\
\hline Renal cell carcinoma & $23 \quad(12)$ & $19(13.9)$ & $3(11.5)$ & $1 \quad(3.5)$ \\
\hline Others & $13(6.8)$ & $6 \quad(4.4)$ & $4(15.4)$ & $3(10.3)$ \\
\hline \multicolumn{5}{|l|}{ Baseline bone metastases (\%) } \\
\hline No & $124(64.6)$ & $101(73.7)$ & $8(30.8)$ & $15(51.7)$ \\
\hline Yes & $68(35.4)$ & $36(26.3)$ & $18(69.2)$ & $14(48.3)$ \\
\hline \multicolumn{5}{|l|}{ Baseline CNS metastases (\%) } \\
\hline No & $162(84.4)$ & $120(87.6)$ & $22(84.6)$ & $20 \quad(70)$ \\
\hline Yes & $30(15.6)$ & $17(12.4)$ & $4(15.4)$ & $9 \quad(30)$ \\
\hline \multicolumn{5}{|l|}{ Anti-PD-1/PD-L1 (\%) } \\
\hline Pembrolizumab & $23 \quad(12)$ & $19(13.9)$ & $2(7.7)$ & $2(6.9)$ \\
\hline Nivolumab & $154(80.2)$ & $110(80.3)$ & $20(76.9)$ & $24(82.8)$ \\
\hline Atezolizumab & $12(6.2)$ & $5 \quad(3.6)$ & $4(15.4)$ & $3(10.3)$ \\
\hline Avelumab & 3 (1.6) & $3 \quad(2.2)$ & - & - \\
\hline \multicolumn{5}{|l|}{ Line of immunotherapy $(\%)$} \\
\hline First line & $30(15.6)$ & $26(19)$ & $1(3.8)$ & $3(10.3)$ \\
\hline Second or subsequent line & $162(84.4)$ & $111(81)$ & $25(96.2)$ & $26(89.7)$ \\
\hline
\end{tabular}

RT, radiotherapy; pRT, palliative RT; ECOG PS, Eastern Cooperative Oncology Group Performance Status; NSCLC, non-small cell lung cancer; CNS, central nervous system; No RT, did not receive RT prior to or during immunotherapy; Previous RT, received pRT within 6 months prior to initiation of immunotherapy; Concurrent $\mathrm{pRT}$, patients who received $\mathrm{pRT}$ during immunotherapy.

whereas ECOG PS and baseline bone metastases were significantly associated with OS (Table III).

The median PFS of the no RT group was 5.4 months (95\% CI, 3.4-7.6), compared with 1.8 months (95\% CI, 1.7-4.1) in the previous pRT group (Fig. 1B) and the difference was significant $(\mathrm{HR}=2.06$; 95\% $\mathrm{CI}, 1.31-3.24 ; \mathrm{P}=0.0016)$. The statistical significance of this difference was not confirmed in the multivariate analysis $(\mathrm{HR}=1.58 ; 95 \% \mathrm{CI}, 0.94-2.65$; $\mathrm{P}=0.0810$ ). Baseline bone metastases and ECOG PS were significantly associated with PFS in the univariate and multivariate analysis, whereas CNS metastases were not (Table SII).

The median TTF of the no RT group was 5.7 months (95\% CI, 3.7-7.4) compared with 1.8 months (95\% CI, 1.3-4.0) of the previous pRT group (Fig. 1C) and this difference resulted in being statistically significant in both the univariate $(\mathrm{HR}=1.92 ; 95 \% \mathrm{CI}, 1.23-2.98 ; \mathrm{P}=0.0035)$ and multivariate analyses ( $\mathrm{HR}=1.76 ; 95 \% \mathrm{CI}, 1.12-2.77 ; \mathrm{P}=0.0132)$. Baseline CNS metastases and bone metastases were not significantly associated with TTF in the multivariate analysis (Table SIII).

Primary tumor (NSCLC vs. others), sex, age and treatment line, were confirmed in the multivariate analyses as significantly associated with OS, DCR, PFS and TTF (Tables SI-IV and III).

In the previous pRT group, $26.9 \%$ of patients experienced irAEs of any grade, compared with $40.1 \%$ of patients in the no RT group. The difference, which may be clinically meaningful, was not statistically significant.

Comparisons between the previous $p R T$ and concurrent $p R T$ groups. ORR and DCR in the concurrent pRT group were $17.9 \%$ (95\% CI, 5.8-41.6) and 42.9\% (95\% CI, 22.1-74.8), respectively. There were no statistically significant differences with the ORR and DCR rates of the previous pRT group 
Table II. Characteristics of patients who received RT.

\begin{tabular}{lcc}
\hline Characteristics & $\begin{array}{c}\text { Previous } \\
\text { pRT, n=26 }\end{array}$ & $\begin{array}{c}\text { Concurrent } \\
\text { pRT, n=29 }\end{array}$ \\
\hline $\begin{array}{l}\text { Total number of pRT treatments } \\
\text { Body site (\%) }\end{array}$ & 27 & 36 \\
CNS & $4(14.8)$ & $7(19.4)$ \\
Bone & $15(55.6)$ & $21(58.4)$ \\
Lymph nodes & $2(7.4)$ & $1(2.8)$ \\
Visceral & $4(14.8)$ & $7(19.4)$ \\
Others & $2(7.4)$ & - \\
Dose of RT, Gy & & \\
$\quad$ Median & 20 & 8 \\
Mean & 23 & 14 \\
Range & $8-40$ & $8-40$ \\
\hline
\end{tabular}

RT, radiotherapy; pRT, palliative RT; CNS, central nervous system; Previous RT, received pRT within 6 months prior to initiation of immunotherapy; Concurrent pRT, patients who received pRT during immunotherapy.

(18.2 and $18.2 \%$, respectively; $\mathrm{P}=1.0000$ and $\mathrm{P}=0.0761$, respectively) (data not shown).

The median OS of the concurrent pRT group was 8.5 months (95\% CI, 5.5-12.4), whereas the median OS of the previous pRT group was 3.6 months (HR=1.31; 95\% CI, 0.71-2.41; $\mathrm{P}=0.3742$ ) and the difference was not significant (Fig. S1A).

The median PFS of the concurrent pRT group was 5.0 months (95\% CI, 3.3-7.2), and in the previous pRT group, the median PFS was 1.8 months (HR=1.58; 95\% CI, 0.91-2.74; $\mathrm{P}=0.1045$ ) and the difference was not significant (Fig. S1B).

The median TTF of the concurrent pRT group was 5.8 months $(95 \%$ CI, 4.1-10.4) compared with 1.8 months (95\% CI, 1.3-4.0) of the previous pRT. The difference was significant in both the univariate (HR=1.99; 95\% CI, 1.12-3.53; $\mathrm{P}=0.0187)$ and multivariate analyses $(\mathrm{HR}=2.42$; 95\% CI, 1.32-4.42; $\mathrm{P}=0.0040$ ) (Fig. S1C; Table SIV).

In the concurrent pRT group, 5 out of 29 patients (17.2\%) experienced irAEs of any grade although this was not significantly lower compared with the $26.9 \%$ incidence rate in the previous $\mathrm{pRT}$ group $(\mathrm{P}=0.3898)$ (data not shown).

Finally, the concurrent pRT and no RT groups were compared (data not shown), and similar results were observed for ORR (17.9 vs. $32 \%$; $\mathrm{P}=0.1316$ ), DCR (42.9 vs. $52.8 \%$; $\mathrm{P}=0.3445)$, $\mathrm{OS}$ (8.4 months vs. 12.1 months; $\mathrm{P}=0.0578)$ and TTF (5.6 vs. 5.7 months; $\mathrm{P}=0.7501$ ), respectively, although PFS was slightly but significantly increased in the concurrent pRT group (5.0 vs. 5.4 months; $\mathrm{P}=0.0459$ ).

\section{Discussion}

The results of the PRACTICE study suggest a possible negative impact of receiving pRT within 6 months prior to immunotherapy initiation, at least in terms of DCR, but likely also of PFS (3x higher in the no RT group) and OS ( $>3 \mathrm{x}$ higher in the no RT). Beyond the statistical significance, not always confirmed at multivariate analyses (possibly due to the small sample size), the survival differences observed among subgroups may be clinically meaningful. Additionally, there was a trend towards less CKI-related toxicity for patients who received $\mathrm{pRT}$ suggesting lower immune-reactivity in these patients. The irAEs incidence rate was the lowest in the concurrent pRT group, low in the previous pRT group and the highest in the no RT group. This is consistent with the previous studies: The higher the effectiveness, the higher the toxicity of CKIs $(30,31)$. Additionally, these findings also confirm the relative safety of pRT during immunotherapy with CKIs, possibly due to the low median dose (8 Gy in the concurrent pRT group).

The possible negative impact of pRT on immunotherapy effectiveness may be mitigated in the concurrent pRT group, as they exhibited improved TTF periods compared with both the no RT and previous pRT group, which both exhibited similar TTF periods. Thus, it is hypothesized that a positive selection bias of oligo-progressive patients, continuing immunotherapy with clinical indication to loco-regional pRT on a single progressing/painful lesion, may have resulted in the improved TTF periods observed in the concurrent pRT group.

Several studies have demonstrated the immunosuppressive effect of RT (particularly on T lymphocytes, which are considered the most radiosensitive cells of the hematopoietic system), from old preclinical models to more recent clinical studies (32-35). Lymphocyte count has been demonstrated to decrease after administration of low-doses of pRT in patients with lung cancer (35). Additionally, radiation-induced reduction of circulating lymphocyte count and eventual lymphocyte infiltration of tumors demonstrated a significant negative impact on OS (32). Radiation is able to activate tumor-growth factors, such as TGF- $\beta$, and to possibly favor tumor progression through the enhancement of M2 macrophages and the increase of T-regulatory cells, which are the most radio-resistant $\mathrm{T}$ cells (8). Thus, taking into account the fact that the abscopal effect is not a common event and is likely dependent on a number of factors, the negative findings of this study agree with our current understanding of palliative RT and immunotherapy.

A major limitation of this retrospective analysis is that the selection of patients receiving $\mathrm{pRT}$ at any time in their clinical history constitutes a selection bias, particularly with the inclusion of subjects likely suffering from bone or CNS metastases, both of which are well-known poor prognostic factors for advanced cancer patients (36-39).

To verify and to reduce the impact of this limitation, a more reliable comparison between the previous pRT and concurrent pRT groups was performed, which were more likely to be affected by bone or CNS metastases with a similar incidence. Moreover, a further analysis to weight the selection bias throughout the direct comparison of patients' characteristics, comparing the incidence of bone and CNS metastases across subgroups was performed.

The incidence of bone metastases at baseline was significantly higher in the previous pRT group compared with both the other groups. The presence of bone metastases at diagnosis is well-known as poor prognostic factor for lung cancer, melanoma and renal cancer (36-38), confirming a probable selection bias in the present study. However, the presence of CNS 

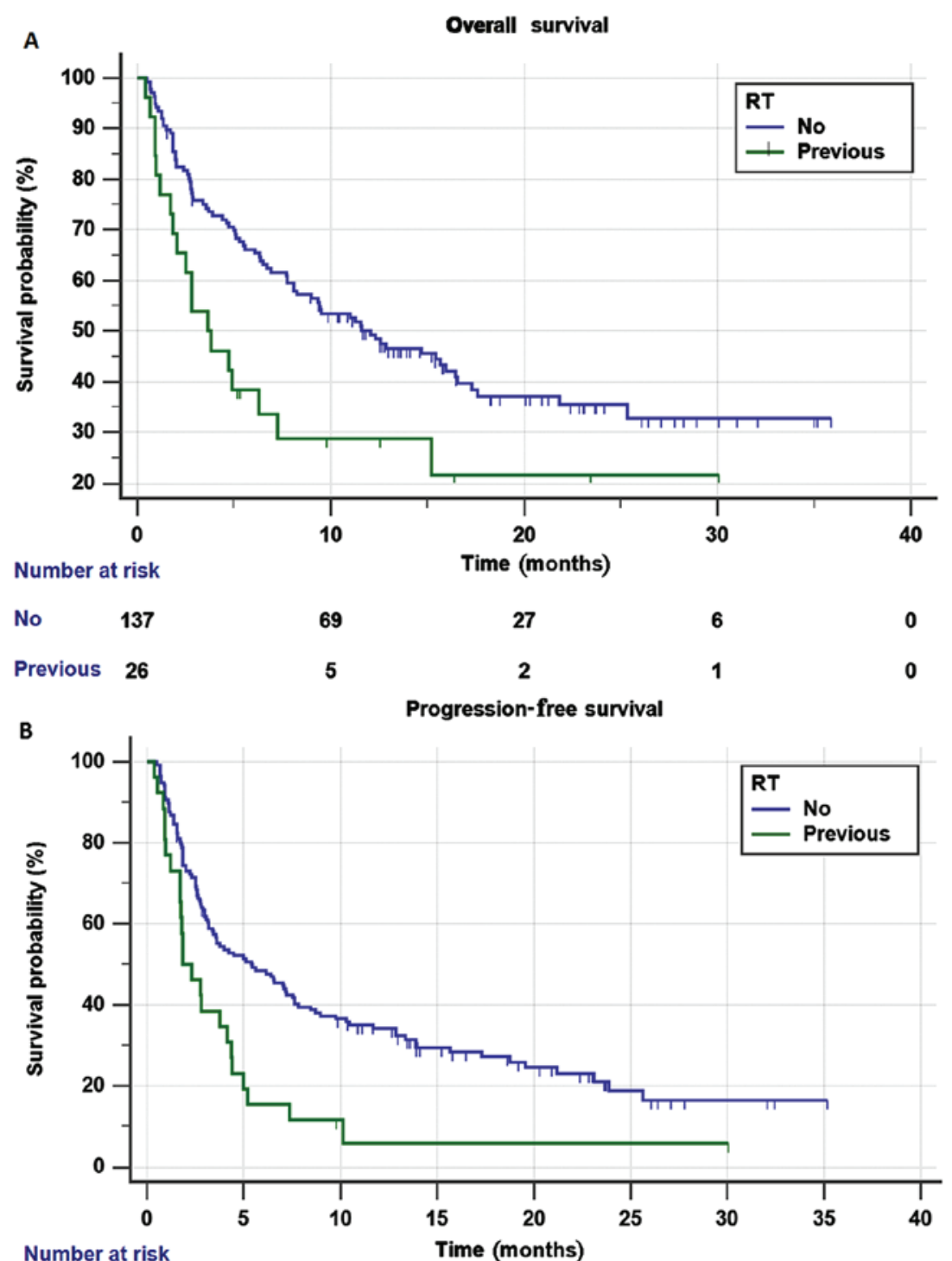

Number at risk

Time (months)
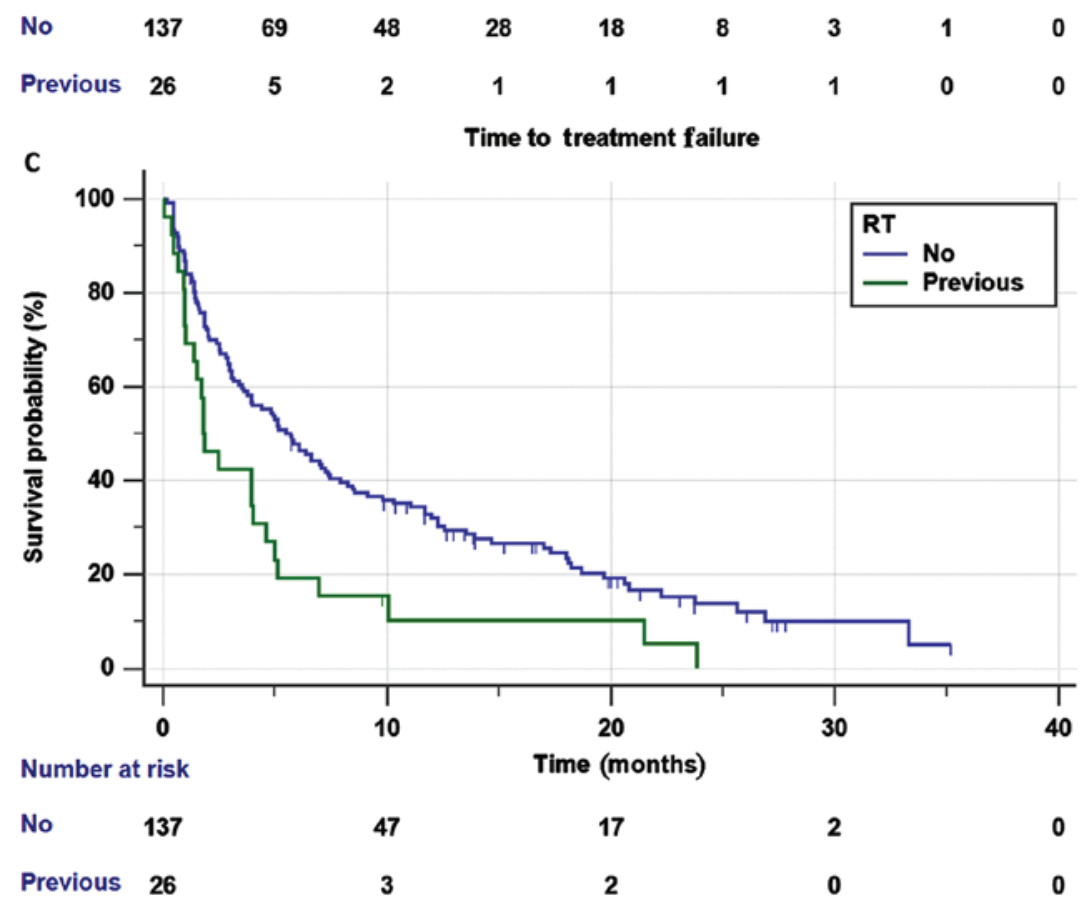

Figure 1. Kaplan-Meier survival curves of patients in the Previous RT group and patients in the no RT group. (A) Overall survival, (B) progression free survival, (C) time to treatment failure. RT, radiotherapy; no RT, did not receive RT prior to or during immunotherapy; Previous RT, received pRT within 6 months prior to initiation of immunotherapy. 
Table III. Univariate and multivariate analysis of overall survival.

\begin{tabular}{llr}
\hline Variables & \multicolumn{1}{c}{$\begin{array}{c}\text { Univariate analysis, } \\
\text { HR }(95 \% \mathrm{CI}) ; \mathrm{P}-\mathrm{value}\end{array}$} & $\begin{array}{c}\text { Multivariate analysis, } \\
\mathrm{HR}(95 \% \mathrm{CI}) ; \mathrm{P}-\mathrm{value}\end{array}$ \\
\hline $\begin{array}{l}\text { Previous pRT, Yes vs. No } \\
\text { Primary tumor, NSCLC vs. }\end{array}$ & $1.94(1.17-3.22) ; \mathrm{P}=0.0095$ & $1.64(0.94-2.86) ; \mathrm{P}=0.0775$ \\
Melanoma & $0.54(0.31-0.97) ; \mathrm{P}=0.0398$ & $0.65(0.36-1.18) ; \mathrm{P}=0.6581$ \\
Kidney & $0.89(0.50-1.59) ; \mathrm{P}=0.7030$ & $1.05(0.58-1.92) ; \mathrm{P}=0.8586$ \\
Others & $1.12(0.45-2.79) ; \mathrm{P}=0.7999$ & $1.10(0.43-2.79) ; \mathrm{P}=0.8399$ \\
Sex, male vs. female & $1.25(0.78-2.01) ; \mathrm{P}=0.3377$ & - \\
Age, $\geq 70$ years old vs. $<70$ years old & $1.61(1.08-2.39) ; \mathrm{P}=0.0184$ & $1.34(0.89-2.04) ; \mathrm{P}=0.1525$ \\
Treatment line, second and subsequent lines vs. first line & $1.75(0.96-3.21) ; \mathrm{P}=0.0673$ & - \\
ECOG PS, $\geq 2$ vs. 0-1 & $3.87(2.53-5.93) ; \mathrm{P}<0.0001$ & $3.82(2.44-5.96) ; \mathrm{P}<0.0001$ \\
Baseline CNS metastases, yes vs. no & $1.17(0.66-2.07) ; \mathrm{P}=0.5750$ & - \\
Baseline bone metastases, yes vs. no & $1.67(1.12-2.49) ; \mathrm{P}=0.0117$ & $1.54(1.01-2.37) ; \mathrm{P}=0.0456$ \\
\hline
\end{tabular}

pRT, palliative radiotherapy; ECOG PS, Eastern Cooperative Oncology Group Performance Status; NSCLC, non-small cell lung cancer; CNS, central nervous system; HR, hazard ratio; CI, confidence interval; Previous RT, received pRT within 6 months prior to initiation of immunotherapy.

metastases was not a further selection bias for the previous pRT group as the incidence was not significantly greater.

The incidence of CNS metastases at baseline were significantly higher in the concurrent pRT group compared with the no RT group and this is a well-known prognostic factor predicting a less favorable outcome (24), it did not result in a clinically meaningful difference in outcome among these two subgroups.

There were no statistically significant differences observed between the previous pRT group and concurrent pRT group in terms of PFS and OS, although this may be the result of the small sample size, potentially clinically meaningful trends were noticed, including a doubling of survival times in the concurrent pRT group. Furthermore, TTF was significantly shorter for patients in the previous pRT group, suggesting that a selection bias did not influence the results, thus highlighting the reliability of the comparison between irradiated and non-irradiated patients.

Taking into account the limitations, the negative selection bias alone likely did not affect the results, as all the other retrospective studies on the impact of RT in patients with advanced cancer treated with CKIs were similarly affected by the same selection bias (12), and the results of these studies almost always show the beneficial effect of receiving RT (9-12), contrasting with the results of the present study.

Comparing the characteristics of patients and of radiation treatments in previous studies, highlighted crucial differences; the type and dose of RT. Frequently, patients reported as receiving 'palliative care' in the literature often received high-dose hypofractioned RT (40), thus preventing confirmation of whether the immunosuppressive effect may be the result of purely palliative RT. To the best of our knowledge, there are no studies examining palliative RT treatments during and before immunotherapy.

In this analysis, the median dose of RT was 20 Gy for the previous pRT group and 8 Gy for the concurrent pRT group, demonstrating that our study population was different from those of previous studies. In the studies suggesting a possible synergy between RT and immunotherapy, stereotactic RT was predominantly used. In other studies reporting positive results, the patient population was heterogeneous, with both palliative and curative radiation approaches (9-12). In the previously cited KEYNOTE-001 sub-analysis (12), comparing RT prior to immunotherapy with no RT, $36 \%$ of patients received definitive (curative) RT (stereotactic body RT or SRS). The weight of such a subgroup could have shifted the balance of the final impact in favor of the abscopal effect of RT, instead of the immunosuppressive one, explaining the positive results of that study. This happens despite a possible selection bias, in fact, in such a study, patients with previous RT had a significantly greater frequency of brain metastases (data concerning bone metastases were not reported) (12).

Therefore, it can be hypothesized that, irrespective of the clear limitations of the present and previous retrospective studies, there are differences in the immunomodulatory effects of different RT approaches, with high-dose, low-volume irradiations providing more favorable results compared with lower-dose, purely palliative RT treatments.

Additionally, the site of irradiation is likely to affect the value of RT. For example a previous study included cases of definitive thoracic RT on pulmonary or nodal lesions (12), whereas in another study, patients receiving bone RT accounted for $>50 \%$ of cases, thus it may be the case that the abscopal effect may be more readily initiated by irradiating soft lesions, in which the immune-infiltrating context would be more conspicuous, offering greater probability of containing antigen-presenting cells (11).

Interpretation of the present study suggests a negative shift in the balance between favorable and unfavorable immune-modulating effects of RT in the case of pRT, a hypothesis that is presented in Fig. 2.

The limitations of the present analysis, with the use of a retrospective cohort, the presence of a selection bias and 


\section{Possible immunomodulatory effects of Radiotherapy}

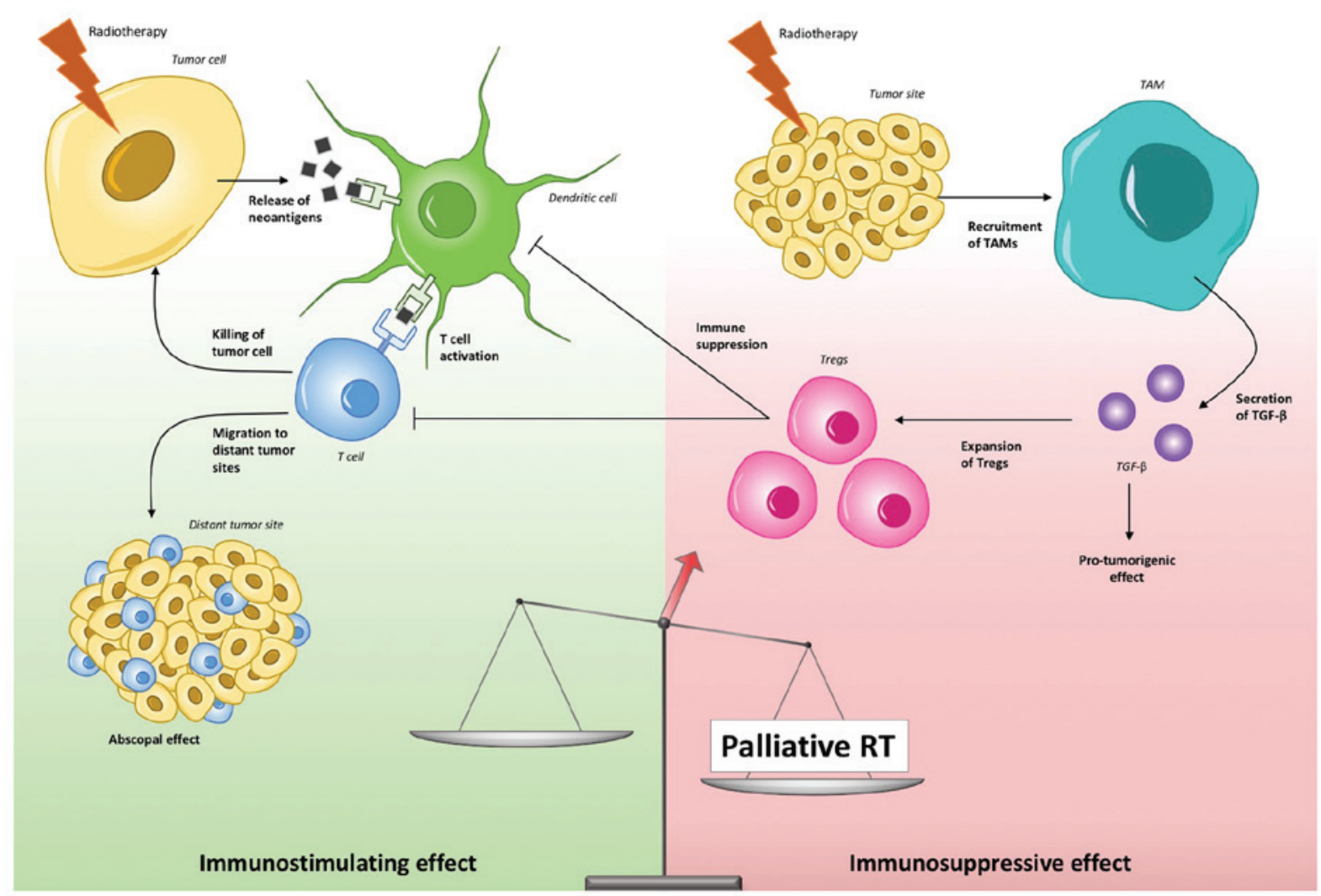

Figure 2. Possible immunomodulatory effects of radiotherapy. TAM, tumor-associated macrophage; TGF- $\beta$, tumor growth factor $\beta$; Tregs, regulatory T cells; $\mathrm{RT}$, radiotherapy.

the small size of the groups receiving pRT, in addition to the lack of details regarding RT volumes and techniques, prevent conclusions from being drawn regarding the use of pRT during immunotherapy. Nevertheless, the present study highlights the need for future prospective analysis to determine the clinical efficacy of pRT by stratifying the population based on RT dose, fractioning, planning and timing in relation to immunotherapy.

\section{Acknowledgements}

We would like to thank the Consorzio Interuniversitario Nazionale per la Bio-Oncologia for their support in this study.

\section{Funding}

No funding was received.

\section{Availability of data and materials}

The datasets used during the present study are available from the corresponding author upon reasonable request.

\section{Authors' contributions}

$\mathrm{MB}$ and $\mathrm{AC}$ conceived and designed the study and drafted the manuscript. MB, EL, NDA, SB, AL, MGC, ST, GG, GP, KC,
PDM, AG, NT, MDT, EG, MM, PB, FP, LC, MT, DG, CN, CF, MT and AC acquired the data. MB, SB, MT, AC and NDA analyzed and interpreted the data. SB, MT, NDA, CN, DG, GG and CF critically revised the manuscript. All authors read and approved the manuscript. All authors have agreed both to be personally accountable for the author's own contributions and to ensure that questions related to the accuracy or integrity of any part of the work, even ones in which the author was not personally involved, are appropriately investigated, resolved, and the resolution documented in the literature.

\section{Ethics approval and consent to participate}

All patients provided written, informed consent for treatment with immunotherapy. The present study was approved by the respective Ethical Committees on Human Experimentation of each institute, after previous approval by the coordinating center (University of L'Aquila, Via Vetoio, Italy; approval no. 32865, approved on July 24th, 2018).

\section{Patient consent for publication}

Not applicable.

\section{Competing interests}

The authors declare that they have no competing interests. 


\section{References}

1. Chung C: To do or not to do: A concise update of current clinical controversies in immune checkpoint blockade. J Oncol Pharm Pract 25: 663-673, 2018.

2. Derosa L, Hellmann MD, Spaziano M, Halpenny D, Fidelle M, Rizvi H, Long N, Plodkowski AJ, Arbour KC, Chaft JE, et al Negative association of antibiotics on clinical activity of immune checkpoint inhibitors in patients with advanced renal cell and non-small-cell lung cancer. Ann Oncol 29: 1437-1444, 2018.

3. Garant A, Guilbault C, Ekmekjian T, Greenwald Z, Murgoi P and Vuong T: Concomitant use of corticosteroids and immune checkpoint inhibitors in patients with hematologic or solid neoplasms: A systematic review. Crit Rev Oncol Hematol 120: 86-92, 2017.

4. Bersanelli M, Giannarelli D, Castrignanò P, Fornarini G, Panni S, Mazzoni F, Tiseo M, Rossetti S, Gambale E, Rossi E, et al: INfluenza vaccine indication during therapy with immune checkpoint inhibitors: A transversal challenge. The INVIDIa study. Immunotherapy 10: 1229-1239, 2018.

5. Lesueur P, Escande A, Thariat J, Vauléon E, Monnet I, Cortot A, Lerouge D, Danhier S, Dô P, Dubos-Arvis C, et al: Safety of combined PD-1 pathway inhibition and radiation therapy for non-small-cell lung cancer: A multicentric retrospective study from the GFPC. Cancer Med 7: 5505-5513, 2018.

6. Liu Y, Dong Y, Kong L, Shi F, Zhu H and Yu J: Abscopal effect of radiotherapy combined with immune checkpoint inhibitors. J Hematol Oncol 11: 104, 2018.

7. Nobler MP: The abscopal effect in malignant lymphoma and its relationship to lymphocyte circulation. Radiology 93: 410-412, 1969.

8. Formenti SC and Demaria S: Combining radiotherapy and cancer immunotherapy: A paradigm shift. J Natl Cancer Inst 105: 256-265, 2013.

9. Kiess AP, Wolchok JD, Barker CA, Postow MA, Tabar V, Huse JT, Chan TA, Yamada Y and Beal K: Stereotactic radiosurgery for melanoma brain metastases in patients receiving ipilimumab: Safety profile and efficacy of combined treatment. Int J Radiat Oncol Biol Phys 92: 368-375, 2015.

10. Lehrer EJ, Peterson J, Brown PD, Sheehan JP, QuiñonesHinojosa A, Zaorsky NG and Trifiletti DM: Treatment of brain metastases with stereotactic radiosurgery and immune checkpoint inhibitors: An international meta-analysis of individual patient data. Radiother Oncol: 104-112, 2019.

11. Theelen WSME, Peulen HMU, Lalezari F, van der Noort V, de Vries JF, Aerts JGJV, Dumoulin DW, Bahce I, Niemeijer AN, de Langen AJ, et al: Effect of pembrolizumab after stereotactic body radiotherapy vs pembrolizumab alone on tumor response in patients with advanced non-small cell lung cancer: Results of the PEMBRO-RT phase 2 randomized clinical trial. JAMA Oncol 5: 1276-1282 2019.

12. Shaverdian N, Lisberg AE, Bornazyan K, Veruttipong D, Goldman JW, Formenti SC, Garon EB and Lee P: Previous radiotherapy and the clinical activity and toxicity of pembrolizumab in the treatment of non-small-cell lung cancer: A secondary analysis of the KEYNOTE-001 phase 1 trial. Lancet Oncol 18: 895-903, 2017.

13. Eisenhauer EA, Therasse P, Bogaerts J, Schwartz LH, Sargent D, Ford R, Dancey J, Arbuck S, Gwyther S, Mooney M, et al: New response evaluation criteria in solid tumours: Revised RECIST guideline (version 1.1). Eur J Cancer 45: 228-247, 2009.

14. Ciocan D, Barbe C, Aubin F, Granel-Brocard F, Lipsker D, Velten M, Dalac S, Truchetet F, Michel C, Mitschler A, et al: Distinctive features of melanoma and its management in elderly patients: A population-based study in France. JAMA Dermatol 149: 1150-1157, 2013.

15. Gridelli C, Balducci L, Ciardiello F, Di Maio M, Felip E, Langer C, Lilenbaum RC, Perrone F, Senan S and de Marinis F: Treatment of elderly patients with non-small cell lung cancer: Results of an international expert panel meeting of the Italian association of thoracic oncology. Clin Lung Cancer 16: 325-333, 2015.

16. Azawi NH, Joergensen SM, Jensen NV, Clark PE and Lund L; Academy of Geriatric Cancer Research (AgeCare): Trends in Kidney cancer among the elderly in Denmark, 1980-2012. Acta Oncol 55 (Suppl 1): S79-S84, 2016.

17. Datta SS, Ghosal N, Daruvala R, Chakraborty S, Shrimali RK, van Zanten C, Parry J, Agrawal S, Atreya S, Sinha S, et al: How do clinicians rate patient's performance status using the ECOG performance scale? A mixed-methods exploration of variability in decision-making in oncology. Ecancermedicalscience 13: 913, 2019.
18. Common terminology criteria for adverse events (ctcae) version 5.0. https://ctep.cancer.gov/protocoldevelopment/electronic_applications/docs/CTCAE_v5_Quick_Reference_8.5x11. pdf. Accessed November 27, 2017.

19. Walle T, Martinez Monge R, Cerwenka A, Ajona D, Melero I and Lecanda F: Radiation effects on antitumor immune responses: Current perspectives and challenges. Ther Adv Med Oncol 10: 1758834017742575,2018

20. Verastegui EL, Morales RB, Barrera-Franco JL, Poitevin AC and Hadden J: Long-term immune dysfunction after radiotherapy to the head and neck area. Int Immunopharmacol 3: 1093-1104, 2003.

21. Mantel N: Chi-square tests with one degree of freedom; extensions of the Mantel-Haenszel procedure. J Am Stat Assoc 58: 690-700, 1963

22. Fisher RA: On the interpretation of $\chi^{2}$ from contingency tables, and the calculation of P. J Royal Stat Soc 85: 87-94, 1992.

23. Mosteller F: Association and estimation in contingency tables. J Am Stat Assoc 63: 1-28, 1968.

24. Cagney DN, Martin AM, Catalano PJ, Redig AJ, Lin NU, Lee EQ, Wen PY, Dunn IF, Bi WL, Weiss SE, et al: Incidence and prognosis of patients with brain metastases at diagnosis of systemic malignancy: A population-based study. Neuro Oncol 19: 1511-1521, 2017.

25. Gdowski AS, Ranjan A and Vishwanatha JK: Current concepts in bone metastasis, contemporary therapeutic strategies and ongoing clinical trials. J Exp Clin Cancer Res 36: 108, 2017.

26. Hosmer DW Jr, Lemeshow S and Sturdivant RX: Applied Logistic Regression. $3^{\text {rd }}$ edition. John Wiley \& Sons (eds). Hoboken, NJ, 2013. https://doi.org/10.1002/9781118548387.

27. Kaplan EL and Meier P: Nonparametric estimation from incomplete observations. J Am Stat Assoc 53: 457-481, 1958.

28. Schemper M and Smith TL: A note on quantifying follow-up in studies of failure time. Control Clin Trials 17: 343-346, 1996.

29. Cox DR: Regression models and life tables. J Royal Stat Soc B (Method) 34: 187-220, 1972.

30. Freeman-Keller M, Kim Y, Cronin H, Richards A, Gibney G and Weber JS: Nivolumab in resected and unresectable metastatic melanoma: Characteristics of immune-related adverse events and association with outcomes. Clin Cancer Res 22: 886-894, 2016.

31. Haratani K, Hayashi H, Chiba Y, Kudo K, Yonesaka K, Kato R, Kaneda H, Hasegawa Y, Tanaka K, Takeda M and Nakagawa K: Association of immune-related adverse events with nivolumab efficacy in non-small-cell lung cancer. JAMA Oncol 4: 374-378, 2018.

32. McDermott CE and Gengozian N: The effect of low exposure-rate gamma irradiation on $\mathrm{T}$ and $\mathrm{B}$ lymphocyte function in the mouse. Int J Radiat Biol Relat Stud Phys Chem Med 37: 415-428, 1980.

33. Gerber M, Pioch Y, Dubois JB and Serrou B: Effects of low doses of irradiation on the T-cell-mediated cytotoxic response. Ann Immunol (Paris) 134C: 149-157, 1983.

34. Venkatesulu BP, Mallick S, Lin SH and Krishnan S: A systematic review of the influence of radiation-induced lymphopenia on survival outcomes in solid tumors. Crit Rev Oncol Hematol 123: 42-51, 2018.

35. Wolny-Rokicka E, Brzeźniakiewicz-Janus K, Wydmański J, Tukiendorf A and Zembroń-Łacny A: Analysis of haemostasis biomarkers in patients with advanced stage lung cancer during hypofractionated radiotherapy treatment. J Int Med Res 46: $1876-1883,2018$

36. Fujimoto D, Ueda H, Shimizu R, Kato R, Otoshi T, Kawamura T, Tamai K, Shibata Y, Matsumoto T, Nagata K, et al: Features and prognostic impact of distant metastasis in patients with stage IV lung adenocarcinoma harboring EGFR mutations: Importance of bone metastasis. Clin Exp Metastasis 31: 543-551, 2014.

37. Barth A, Wanek LA and Morton DL: Prognostic factors in 1,521 melanoma patients with distant metastases. J Am Coll Surg 181: 193-201, 1995.

38. Ruatta F, Derosa L, Escudier B, Colomba E, Guida A Baciarello G, Loriot Y, Fizazi K and Albiges L: Prognosis of renal cell carcinoma with bone metastases: Experience from a large cancer centre. Eur J Cancer 107: 79-85, 2019.

39. Lee EQ: Nervous system metastases from systemic cancer. Continuum (Minneap Minn) 21: 415-428, 2015.

40. Aboudaram A, Modesto A, Chaltiel L, Gomez-Roca C, Boulinguez S, Sibaud V, Delord JP, Chira C, Delannes M, Moyal E and Meyer N: Concurrent radiotherapy for patients with metastatic melanoma and receiving anti-programmed-death 1 therapy: A safe and effective combination. Melanoma Res 27: 485-491, 2017.

This work is licensed under a Creative Commons Attribution-NonCommercial-NoDerivatives 4.0 International (CC BY-NC-ND 4.0) License. 